\title{
四嗪与一些环烯烃反应机理的理论研究
}

\author{
方德彩* 陈彦梅 \\ (北京师范大学化学学院 北京 100875)
}

\begin{abstract}
摘要 采用多种密度泛函方法并辅以极化连续介质模型研究了四嗪与一些环烯烃发生[4+2]环加成反应的反应机理, 得出反应过程按协同机理进行，即反应过程中只有一个过渡态. 主要使用的方法有: CAM-B3LYP, B3LYP, X3LYP, BMK, LC-wPBE, wB97x, wB97xd, M062x 和 M11. 这种过渡态的稳定性与前线分子轨道的相互作用有关, 从过渡态电 荷迁移的方向来看，环烯烃作为电子给体，而四嗪作为电子受体. 反应的难易取决于环烯烃的环的大小，环越大反应 越难, 这与环在形成过渡态时的变形能有关. 通过气相平动熵和溶液平动熵计算得到的活化自由能的数据, 比较发现 气相平动能得出的活化自由能离实验估计值相差很远. 而对于溶液中活化自由能垒数据对于大多数计算方法所得的结 果比较接近, 其中 BMK, CAM-B3LYP 和 X3LYP 的结果更合理一些. 同时也发现 M062x 和 M11 方法计算的反应速度 常数与实验值差别较大, 说明这些方法不太适合用来研究此类反应; 而考虑色散作用的 wB97xd 也过高估计过渡态时 分子之间的作用能，导致低自由能垒，具体产生的原因在文中进行了详细的讨论.
\end{abstract}

关键词 四嗪; 环烯烃; [4+2]环加成; 溶液中自由能; 反应速度常数

\section{Theoretical Studies on the Mechanism of Cycloaddition Reaction between 1,2,4,5-Tetrazine and Cycloolefines}

\author{
Fang, Decai* Chen, Yanmei \\ (College of Chemistry, Beijing Normal University, Beijing 100875)
}

\begin{abstract}
The reaction mechanism of [4+2] cycloaddition reaction between 1,2,4,5-tetrazine and cycloolefines has been investigated with several density functional theory (DFT) methods, such as CAM-B3LYP, B3LYP, X3LYP, BMK, LC-wPBE, wB97x, wB97xd, M062x and M11, and the obtained results show that all of title reactions are concerted regardless of methods used. The stability of transition state is moderated by the interaction of frontier molecular orbitals (FMO), in which cycloolefine acts as an electron-donating partner and 1,2,4,5-tetrazine acts as an electron-accepter from the charge transfer direction in the transition state. The calculated activation free-energy barrier is dependent on the size of ring in cycloolefines, i.e., the larger the size of ring in cycloolefine, the more difficult the reaction, which is related to the deformation energy when transition state is formed. The activation free energy barriers have been calculated both with gas-phase translational entropy and solution translational entropy, in which those from gas-phase translational entropy (output of Gaussian job) are far away from the experimental estimations. For those generated from solution translational entropy are comparable for most of DFT methods, in which those generated from BMK, CAM-B3LYP and X3LYP methods are more reasonable. However, rate constants calculated from both M062x and M11 methods are largely deviated from experimental observations, indicating that these two methods are not suitable for studying these types of reactions. Moreover, the wB97xd method with classical dispersion overestimates the interaction between two fragments in transition states, and thus leads to low activation free energy barrier, meaning that those transition states, stabilized by FMO interactions, are hardly to form temperate and regional dipoles for the interacting partners. The reason for the failure of these methods has been addressed in this paper.

Keywords 1,2,4,5-tetrazine; cycloolefine; [4+2] cycloaddition; free-energy in solution; rate constant
\end{abstract}

\section{1 引言}

狄尔斯-阿尔德(Diels-Alder)反应 ${ }^{[1]}$ 是一种由共轭双 烯与取代烯烃参与的环加成反应生成取代环己烯. 大多 数的 Diels-Alder [4+2] 反应是以环成二烯作为双烯参与 反应，如环戊二烯与环丙烯 ${ }^{[2,3]}$ 、环丁烯 ${ }^{[4]}$ 以及环戊烯 ${ }^{[5]}$
等反应，其中环丙烯参与的反应一般在低温下就能进 行 ${ }^{[6,7]}$, 并且已经应用到实际的合成中 ${ }^{[8]}$. 随着环烯烃的 环增大, [4+2]反应越来越难, 对于这种现象, Houk 研究 组 ${ }^{[9]}$ 认为这是与形成过渡态时环的变形能有关. 在过渡 态时, 环丙烯部分的变形能最少, 所以反应最容易. 环

* E-mail: dcfang@bnu.edu.cn; Tel.: 010-58805422; Fax: 010-58802075

Received December 2, 2013; published January 1, 2014.

Supporting information for this article is available free of charge via the Internet at http://sioc-journal.cn.

Project supported by the National Natural Science Foundation of China (No. 21373030).

项目受国家自然科学基金(No. 21373030)资助. 
戊二烯或 1,3-丁二烯与烯烃的反应是理论上研究 Diels-Alder 反应机理的典型例子, 此类反应一般只有一 个过渡态, 即协同反应机理 ${ }^{[9 \sim 16]}$. 而对于四嗪参与的 Diels-Alder 反应研究不多 ${ }^{[9,17]}$, 文献中有关生物分子结 合自由能的计算多采用分子动力学模拟方法得到 ${ }^{[18 \sim 20]}$, 本工作试图用不同的密度泛函方法来研究如图 1 所示的 反应, 以探讨远程相互作用、色散等对反应活化自由能 的影响, 同时也以此反应为例来探讨我们提出的溶液中 自由能计算方法的合理性.

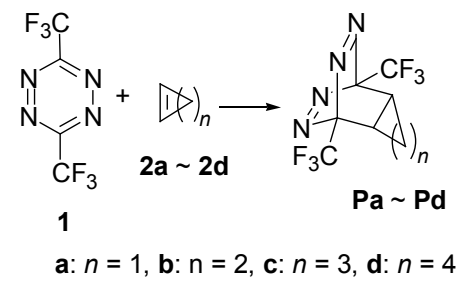

图 1 研究的反应

Figure 1 The studied reactions

\section{2 结果与讨论}

\section{1 反应机理}

对于反应 $\mathrm{a} \sim \mathrm{d}$, 我们分别优化出了反应物、过渡态 和产物的几何构型, 图 2 给出了 4 个反应的过渡态的主 要结构参数, 即两个即将生成的键参数(而具体的直角 坐标见补充材料). 从图中可以看出, 前三个过渡态是 对称的结构, 即这两个键的参数基本上是一样的, 而最 后一个过渡态 TSd 的两个键由于环的不对称而有所不 同, 但是差别也不太大. 从 $\mathrm{TSa}$ 到 $\mathrm{TSc}$, 这两个键的键 长依次变短, 即对于环丙烯和四嗪的反应体系, 在过渡 态时这些键的键长大于 $2.32 \AA$; 而对于环丁烯和四嗪的 反应体系, 其键长在 $2.25 \sim 2.30 \AA$ 之间, 说明前者的过

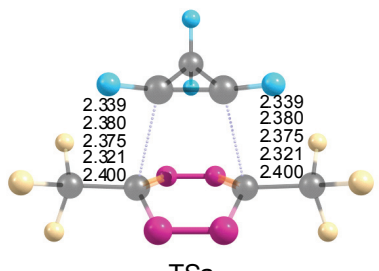

TSa

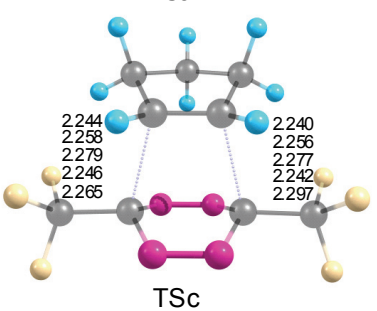

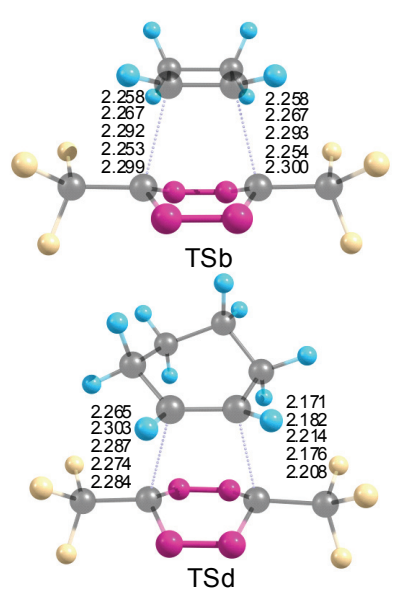

TSd
图 2 主要生成键的几何构型(单位： $\AA$; 从上至下依次为: CAM-B3LYP, BMK, LC-wPBE, wB97 和 M062x)

Figure 2 The main geometric parameters (unit: $\AA$; CAM-V3LYP, BMK, LC-wPBE, wB97 $x$ and M062x, from top to bottom)
渡态比后者早. 不同的方法优化出来的结构参数比较接 近, 最大的差别在 TSa 结构上, wB97x 和 M062x 得到的 结构差别达到 $0.08 \AA$.

从前线轨道来看(图 3), 反应物 1 的 HOMO 和 LUMO 轨道都不参与作用, 因为它们与参与成键的两 个碳原子无关. 从轨道对称性匹配来说, 有两组作用存 在, 如分子 $\mathbf{2 a}$ 的 $\mathrm{HOMO}$ 轨道与分子 $\mathbf{1}$ 的 $\mathrm{LUMO}+1$ 轨 道, 以及分子 1 的 $\mathrm{HOMO}-1$ 轨道与分子 $\mathbf{2 a}$ 的 LUMO +2 轨道, 其中后者由于轨道能级差大, 所以贡献很少, 而前者的能级差小，对称性匹配，所以对过渡态的稳定 性贡献大, 这与在过渡态时有 0.42 个电子从环丙烯转移 到反应物 1 片段上的结果一致. 而对于其它三个反应情 况类似, 轨道能级差也很接近, 在这里不再细述了.

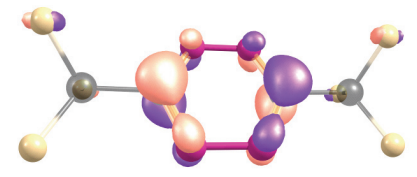

$1 \operatorname{HOMO}-1(e=-12.3 \mathrm{eV})$

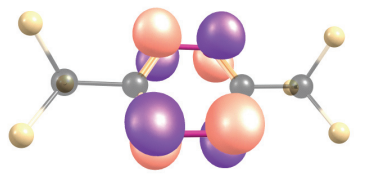

$1 \operatorname{LUMO}(e=-3.0 \mathrm{eV})$

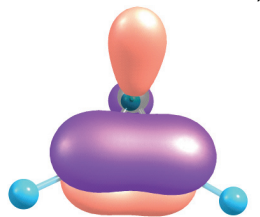

2a $\operatorname{HOMO}(e=-8.6 \mathrm{eV})$

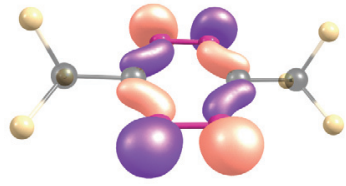

$1 \mathrm{HOMO}(e=-9.8 \mathrm{eV})$

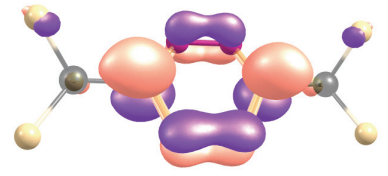

$1 \operatorname{LUMO}+1(e=-2.2 \mathrm{eV})$

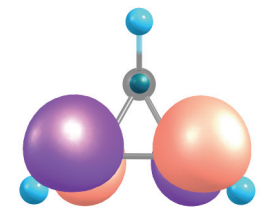

2a LUMO+2 $(e=1.0 \mathrm{eV})$
图 3 反应物 1 和 $2 \mathrm{a}$ 的前线分子轨道及能量

Figure 3 Frontier orbitals and their energies for $\mathbf{1}$ and 2a

\section{2 不同方法下活化自由能的比较}

表 1 给出了四个反应在 $293 \mathrm{~K}$ 下的活化焓垒和活化 自由能垒, 从得到的数据来看, 各种方法都能预测随着 烯烃环的增大，反应变得越来越难，而由气相平动熵计 算出来的自由能(Gaussian 程序直接得到的)的活化自由 能垒偏离实验估计值都很远, 这是因为在溶液中的分子 的熵要比气相中的熵小. 根据 Trouton 规则 ${ }^{[21]}$, 对于大 多数有机化合物来说, 这个差值约为 $-21 \mathrm{cal} \cdot \mathrm{mol}^{-1} \cdot \mathrm{K}^{-1}$, 转化为对自由能的贡献在 $293 \mathrm{~K}$ 下约为 $6.2 \mathrm{kcal} / \mathrm{mol}$, 而 表中两栏自由能垒的差别在 7.1 7.6 kcal $/ \mathrm{mol}$ 之间, 这 主要来自于气相与溶液的平动熵的差异, 因为转动熵和 振动熵的变化在溶液中可以忽略不计 ${ }^{[22]}$. 从所得的自 由能垒数据来看, 除了 M062x, M11 和 wB97xd 偏离远 以外, 其它各种密度泛函所得的结果都比较接近(小于 4 $\mathrm{kcal} / \mathrm{mol}$ ), 而其中实验数据离 CAM-B3LYP 和 BMK 较 近，一般介于两者之间，CAM-B3LYP 的结果略为偏大， 而 BMK 的结果稍偏小. 而对于分子间排斥比较大的反 
应体系 $\mathrm{d}$, BMK 结果明显优于 CAM-B3LYP 结果, 而 X3LYP 结果与 CAM-B3LYP 的非常接近, 这些都明显 优于 B3LYP 的结果. 而考虑色散作用的 wB97xd 的结果 显然比没有考虑色散的 $w B 97 x$ 结果位垒低, 这是因为 过渡态的稳定性主要取决于前线分子轨道的相互作用, 而由 $\pi$ 电子参与成键的过程产生瞬时偶极的可能性很小, 所以如果加上经典的色散效应的话就可能过高地估计 两个反应物之间的相互作用能, 从而导致计算所得的能 垒偏低, 与实验数据相差较远. 很显然, M062x 和 M11 的结果就不可靠了, 一是其活化焓垒是负的值(分别为 -2.7 和 $-3.2 \mathrm{kcal} / \mathrm{mol}$, 见补充材料); 二是即使使用气 相平动熵计算得到的自由能垒也明显比实验值低, 而最 新的 M11 泛函对此类反应位垒的计算结果更差. 这些 方法计算结果不理想的原因是过高估计了远程相互作 用, 从图 4 可以看出, 用 $\mathrm{M} 062 \mathrm{x}$ 优化出来的络合物两个 环之间的距离为 $2.990 \AA$, 按理两个环之间的作用应该 比较弱, 但是在键鞍点的电子密度还是相当大的 $(0.011$

表 1 不同方法计算得到的活化自由能 $(293 \mathrm{~K}, \mathrm{kcal} / \mathrm{mol})$

Table 1 The activation free-energies obtained by different methods $(293 \mathrm{~K}$, in $\mathrm{kcal} / \mathrm{mol})$

\begin{tabular}{|c|c|c|c|c|c|c|c|c|}
\hline & \multicolumn{2}{|c|}{ Reaction $\mathrm{a}^{a}$} & \multicolumn{2}{|c|}{ Reaction $\mathrm{b}$} & \multicolumn{2}{|c|}{ Reaction c } & \multicolumn{2}{|c|}{ Reaction d } \\
\hline & $\begin{array}{c}\Delta G^{\neq} \\
\left(\text {gas) }{ }^{b}\right.\end{array}$ & $\begin{array}{l}\Delta G^{\neq} \\
(\mathrm{sol})^{c}\end{array}$ & $\begin{array}{l}\Delta G^{\neq} \\
\text {(gas) }\end{array}$ & $\begin{array}{l}\Delta G^{*} \\
\text { (sol) }\end{array}$ & $\begin{array}{l}\Delta G^{\neq} \\
\text {(gas) }\end{array}$ & $\begin{array}{l}\Delta G^{\neq} \\
\text {(sol) }\end{array}$ & $\begin{array}{l}\Delta G^{\neq} \\
\text {(gas) }\end{array}$ & $\begin{array}{l}\Delta G^{ \pm} \\
\text {(sol) }\end{array}$ \\
\hline Best est. ${ }^{d}$ & & 13.3 & & 16.0 & & 18.8 & & 22.1 \\
\hline AM-E & 20.8 & 13.0 & 24.5 & 17.3 & 27.7 & 20.3 & 32.6 & 25.3 \\
\hline 33LYP & 21.7 & 14.4 & 25.9 & 18.5 & 29.7 & 22.1 & 34.2 & 26.6 \\
\hline X3LYP & 21.0 & 13.8 & 24.7 & 17.4 & 28.0 & 20.6 & 32.9 & 25.6 \\
\hline 3MK & 20.3 & 13.1 & 22.9 & 15.6 & 25.7 & 18.4 & 30.3 & 22.7 \\
\hline C-wPB & 19.1 & 12.0 & 22.1 & 14.8 & 24.8 & 17.4 & 29.8 & 22.4 \\
\hline wB97x & 19.1 & 12.0 & 21.8 & 14.5 & 24.8 & 17.4 & 29.3 & 22.0 \\
\hline wB97xd & 16.0 & 8.7 & 17.4 & 10.1 & 20.8 & 13.4 & 24.4 & 16.9 \\
\hline M062x & 11.4 & 4.3 & 13.1 & 5.8 & 15.5 & 8.3 & 20.1 & 12.7 \\
\hline M11 & 10.7 & 3.5 & 11.7 & 4.5 & 13.3 & 6.0 & 17.5 & 10.0 \\
\hline
\end{tabular}

${ }^{a}$ The reaction numbering system given in Figure $1 ;{ }^{b}$ Output from Gaussian program, $101 \mathrm{kPa}, 293 \mathrm{~K} ;{ }^{c}$ Calculated from free volume for $1 \mathrm{M}$ and 293 $\mathrm{K}^{[24]} ; \quad{ }^{d}$ Calculated from experimental rate constants ${ }^{[17]}$.

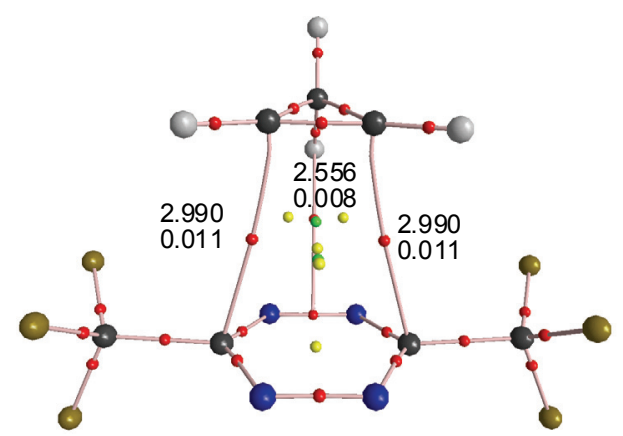

图 4 由 M062x 波函数产生的络合物的分子图(上：键长 $\AA$; 下：电子 密度 au)

Figure 4 Molecular graph for complex between $\mathbf{1}$ and 2a, obtained with M062x wavefunction (top: bond length in $\AA$ and bottom: electron density in au) $\mathrm{au})$, 并且 $\mathrm{M} 062 \mathrm{x}$ 计算得到的稳定化焓在 $293 \mathrm{~K}$ 下为 $-5.9 \mathrm{kcal} / \mathrm{mol}$, 溶液中稳定化自由能为 $-2.4 \mathrm{kcal} / \mathrm{mol}$, 这就意味着这种络合物是稳定的, 很显然这种结果是很 难令人信服的. 近来, Head-Gordon 等 ${ }^{[23]}$ 指出 Truhlar 等 提出的密度泛函家族对于基组重叠误差很难收敛的问 题, 我们认为可能也与此有关.

\section{3 反应速度常数的计算}

表 2 列出了根据过渡状态理论计算得到的四个反应 的速度常数 $(293 \mathrm{~K})$, 并与实验值做对比. 我们知道, 反 应速度常数的计算值对于自由能垒的数据非常敏感，一 般 1 3 kcal/mol 的自由能垒差别就可以产生 5 100 倍 的速度常数差别 $(293 \mathrm{~K})$. 很显然, 由气相平动熵得到的 活化自由能垒计算得到的反应速度常数明显不正确, 有 些结果偏差高达 5 6 个数量级(见补充材料). 而 $\mathrm{M} 062 \mathrm{x}$ 和 M11 得到的数据明显大于实验值, 即使采用气相平 动熵计算的数据也比实验值高 $2 \sim 3$ 个数量级(见补充材 料). 而对于前三个反应, BMK, CAM-B3LYP 和 X3LYP 给出的结果与实验值比较接近, 一般介于 $\mathrm{BMK}$ 和 CAM-B3LYP 及 X3LYP 之间, 但是对于反应 $\mathrm{d}, \mathrm{BMK}$, LC-wPBE 和 wB97x 的结果更合理一些.

表 $2293 \mathrm{~K}$ 时不同方法计算得到的不同反应的速度常数 $\left(\mathrm{M}^{-1} \cdot \mathrm{s}^{-1}\right)$ 的 比较(反应标号见图 1)

Table 2 The comparison for reaction rate constants $\left(\mathrm{M}^{-1} \cdot \mathrm{s}^{-1}\right)$ under 293 $\mathrm{K}$ obtained by different DFT methods (The reaction numbering system given in Figure 1)

\begin{tabular}{lcccc}
\hline & Reaction a & Reaction b & Reaction c & Reaction d \\
\hline Exp. $^{[17]}$ & $7.2 \times 10^{2}$ & 7.4 & $5.6 \times 10^{-2}$ & $1.9 \times 10^{-4}$ \\
CAM-B3LYP & $3.7 \times 10^{2}$ & 0.8 & $3.5 \times 10^{-3}$ & $8.4 \times 10^{-7}$ \\
B3LYP & $1.1 \times 10^{2}$ & 0.1 & $2.0 \times 10^{-4}$ & $9.0 \times 10^{-8}$ \\
X3LYP & $3.1 \times 10^{2}$ & 0.7 & $2.7 \times 10^{-3}$ & $5.0 \times 10^{-7}$ \\
BMK & $1.0 \times 10^{3}$ & 14.3 & $1.2 \times 10^{-1}$ & $0.7 \times 10^{-4}$ \\
LC-wPBE & $6.9 \times 10^{3}$ & 56.5 & $6.5 \times 10^{-1}$ & $1.2 \times 10^{-4}$ \\
wB97x & $6.9 \times 10^{3}$ & 94.6 & $6.5 \times 10^{-1}$ & $2.4 \times 10^{-4}$ \\
wB97xd & $2.0 \times 10^{6}$ & $1.8 \times 10^{5}$ & $6.3 \times 10^{2}$ & 1.5 \\
M062x & $3.8 \times 10^{9}$ & $2.9 \times 10^{8}$ & $4.0 \times 10^{6}$ & $2.1 \times 10^{3}$ \\
M11 & $1.5 \times 10^{10}$ & $2.7 \times 10^{9}$ & $2.1 \times 10^{8}$ & $2.1 \times 10^{5}$ \\
\hline
\end{tabular}

\section{3 结论}

通过对 4 个有关 $[4+2]$ 环加成反应机理的研究, 我 们可以得出以下结论:

(1)此类反应一般是按协同机理进行, 双烯作为电 子受体, 而单烯作为电子给体.

(2)对于考虑经典色散作用的方法如 wB97xd 对于研 究此类反应不太合适, 这是因为色散作用对于这些由前 线分子轨道控制的过渡态应该不明显.

(3)对于 Truhlar 家族的 DFT 方法如 M062x 和 M11, 计算得到的结果不合理, 原因在于过高地估计了反应物 之间的弱相互作用. 而 CAM-B3LYP, BMK 和 X3LYP 


\section{方法是比较适合来研究此类反应的.}

(4)为了得到合理的溶液中反应活化自由能和反应 速度常数, 考虑溶液中平动熵是很有必要的.

\section{4 计算方法}

本工作采用多种密度泛函方法来优化了有关反应 物、过渡态和产物的几何构型, 具体方法如 $\mathrm{B} 3 \mathrm{LYP}^{[25]}$, CAM-B3LYP ${ }^{[26]}, \quad \mathrm{X} 3 \mathrm{LYP}^{[27]}, \mathrm{BMK}^{[28]}, \quad \mathrm{LC}-\mathrm{wPBE}^{[29]}$,

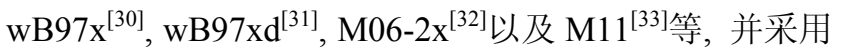
$6-311++\mathrm{G}(\mathrm{d}, \mathrm{p})$ 基组 ${ }^{[34]}$, 所有的计算都采用极化连续介 质模型 $(\mathrm{PCM})^{[35]}$ 和我们的 IDSCRF 半径 ${ }^{[36]}$ 来探讨溶剂 1,4-二氧环已烷的作用. 所有量子化学计算由 Gaussian 09来完成. 自由能的计算采用 Gaussian程序中默认的方 法(气相平动熵)和我们提出的溶液中平动熵两种方法, 这些数据可以由我们自编的 THERMO 程序来计算 ${ }^{[37]}$. 而对于电子密度拓扑分析采用 AIM2000 程序 ${ }^{[38]}$.

\section{References}

[1] Diels, O.; Alder, K. Justus Liebig's Annalen der Chemie 1928, 460, 98.

[2] Wiberg, K. B.; Bartley, W. J. J. J. Am. Chem. Soc. 1960, 82, 6375.

[3] Lou, Y.; Horikawa, M.; Kloster, R. A.; Hawryluk, N. A.; Corey, E. J. J. Am. Chem. Soc. 2004, 126, 8916.

[4] Cadogan, J. I. G.; Cameron, D. K.; Gosney, I.; Tinley, E. J.; Wyse, S. J.; Amaro, A. J. Chem. Soc., Perkin Trans. 1 1991, 2081.

[5] Brúson, H. A.; Riener, T. W. J. Am. Chem. Soc. 1945, 67, 723.

[6] Rubin, M.; Rubina, M.; Gevorgyan, V. Synthesis 2006, 1221.

[7] Zhu, Z.-B.; Wei, Y.; Shi, M. Chem. Soc. Rev. 2011, 40, 5534.

[8] Wilson, M. R.; Taylor, R. E. Angew. Chem., Int. Ed. 2013, 52, 4078.

[9] Liu, F.; Paton, R. S.; Kim, S.; Liang, Y.; Houk, K. N. J. Am. Chem. Soc. 2013, 135, 15642.

[10] Jorgensen, W. L.; Lim, D.; Blake, J. F. J. Am. Chem. Soc. 1993, 115, 2936.

[11] Beno, B. R.; Wilsey, S.; Houk, K. N. J. Am. Chem. Soc. 1999, 121, 4816.
[12] Wilsey, S.; Houk, K. N.; Zewail, A. H. J. Am. Chem. Soc. 1999, 121, 5772.

[13] Diau, E. W.-G.; De Feyter, S.; Zewail, A. H. Chem. Phys. Lett. 1999, 304, 134

[14] Singleton, D. A.; Schulmeier, B. E.; Hang, C.; Thomas, A. A.; Leung, S.-W.; Merrigan, S. R. Tetrahedron 2001, 57, 5149.

[15] Dinadayalane, T. C.; Vijaya, R.; Smitha, A.; Narahari Sastry, G. J. Phys. Chem. A 2002, 106, 1627.

[16] Guner, V.; Khuong, K. S.; Leach, A. G.; Lee, P. S.; Bartberger, M. D.; Houk, K. N. J. Phys. Chem. A 2003, 107, 11445.

[17] Thalhammer, F.; Wallfahrer, U.; Sauer, J. Tetrahedron Lett. 1990, 31,6851 .

[18] Hu, G. D.; Zhang, S. L.; Zhang, Q. G. Acta Chim. Sinica 2009, 67, 1019. (扈国栋, 张少龙, 张庆刚, 化学学报, 2009, 67, 1019.)

[19] Ma, G. Z.; Liu, C.; Qiu, Y. F.; Nan, J. M. Acta Chim. Sinica 2009, 67, 453. (马国正, 刘聪, 求亚芳, 南俊民, 化学学报, 2009, 67, 453.)

[20] Yi, C. H.; Zhang, Q. G. Acta Chim. Sinica 2010, 68, 2029. (伊长虹, 张庆刚, 化学学报, 2010, 68, 2029.)

[21] Gurney, R. W. Introduction to Statistical Mechanics, 1st ed., McGraw-Hill Book Company, New York, 1949.

[22] Frank, H. S. J. Chem. Phys. 1945, 13, 478.

[23] Mardirossian, N.; Head-Gordon, M. J. Chem. Theory Comput. 2013, 9, 4453.

[24] Chen, Y.-M.; Chass, G. A.; Fang, D.-C. Phys. Chem. Chem. Phys. 2014, 16, 1078 .

[25] Lee, C.; Yang, W.; Parr, R. G. Phys. Rev. B 1988, 37, 785.

[26] Yanai, T.; Tew, D.; Handy, N. Chem. Phys. Lett. 2004, 393, 51.

[27] Xu, X.; Goddard, W. A. III. Proc. Natl. Acad. Sci. U. S. A. 2004, 101, 2673.

[28] Boese, A. D.; Martin, J. M. L. J. Chem. Phys. 2004, 121, 3405.

[29] Tawada, Y.; Tsuneda, T.; Yanagisawa, S.; Yanai, T.; Hirao, K. J. Chem. Phys. 2004, 120, 8425.

[30] Chai, J.-D.; Head-Gordon, M. J. Chem. Phys. 2008, 128, 084106.

[31] Chai, J.-D.; Head-Gordon, M. Phys. Chem. Chem. Phys. 2008, 10, 6615.

[32] Zhao, Y.; Truhlar, D. G. J. Phys. Chem. 2006, 110, 5121.

[33] Peverati, R.; Truhlar, D. G. J. Phys. Chem. Lett. 2011, 2, 2810.

[34] McLean, A. D.; Chandler, G. S. J. Chem. Phys. 1980, 72, 5639.

[35] Miertus, S.; Scrocco, E.; Tomasi, J. Chem. Phys. 1981, 55, 117.

[36] Tao, J.-Y.; Mu, W.-H.; Chass, G. A.; Tang, T.-H.; Fang, D.-C. Int. J. Quantum Chem. 2013, 113, 975.

[37] Fang, D.-C. THERMO, Beijing Normal University, Beijing, China.

[38] See http://gauss.fh-bielefeld.de/aim2000. 